\title{
Heart Valve Leaflet Device
}

National Cancer Institute

\section{Source}

National Cancer Institute. Heart Valve Leaflet Device. NCI Thesaurus. Code C50029.

A mechanical heart valve where the valve mechanisms consists of two thin blades that hinge from the center and regulate the flow of blood. 